\title{
Climate Change, Uncertainty and Policy
}

Jeroen Hopster,

University of Graz \& University of Twente; j.k.g.hopster@utwente.nl

\begin{abstract}
While the foundations of climate science and ethics are well established, fine-grained climate predictions, as well as policy-decisions, are beset with uncertainties. This chapter maps climate uncertainties and classifies them as to their ground, extent and location. A typology of uncertainty is presented, centered along the axes of scientific and moral uncertainty. This typology is illustrated with paradigmatic examples of uncertainty in climate science, climate ethics and climate economics. Subsequently, the chapter discusses the IPCC's preferred way of representing uncertainties and evaluates its strengths and weaknesses from a risk management perspective. Three general strategies for decision-makers to cope with climate uncertainty are outlined, the usefulness of which largely depends on whether or not decision-makers find themselves in a context of deep uncertainty. The chapter concludes by offering two recommendations to ease the work of policymakers, faced with the various uncertainties engrained in climate discourse.
\end{abstract}

\section{Keywords}

Climate change; Scientific uncertainty; Moral uncertainty; Deep uncertainty; Risk; IPCC; Storylines; Probability; Expected utility

\section{Introduction}

The role of uncertainty in climate change discourse is a complex and thorny issue. Its complexity is due to the fact that climate uncertainties are of different kinds, interact with each other, and manifest themselves at various locations in climate science, economics and ethics. Its thorniness stems from the track record of interest groups who have appropriated - and manufactured - climate uncertainties for political and ideological purposes, typically to downplay the importance of climate action (Oreskes and Conway 2010).

This chapter focuses on the former issue: it discusses the entangled uncertainties that beset climate discourse and outlines strategies for coping with uncertainty in policymaking. To preclude misappropriation, however, let me preface the discussion by highlighting some of the issues over which very little uncertainty exists within communities of relevant experts (e.g. IPCC 2013):

i) The main physical mechanism that gives rise to global warming is the greenhouse effect, which has been known for a long time and is scientifically well understood. The accumulation of greenhouse gas molecules in the atmosphere causes ever more heat to be retained on Earth, resulting in rising temperature levels, as well as other changes in the Earth's climate.

ii) Anthropogenic emissions of greenhouse gases, specifically of $\mathrm{CO}_{2}$, are the major driver of the climate change we are currently experiencing. Unless global greenhouse gas emissions are rapidly curtailed, the impacts of climate change are very likely to become more severe over the next decades.

iii) Anthropogenic climate change is associated with several risks and hazards, including an increase of weather extremes, droughts, floods, rising sea levels, biodiversity loss and associated risks to food security, human health, water supply, liveability and economic growth.

iv) While some countries might benefit, at least in the short run, from global warming, and while some animal species may thrive in warmer climates, there are clear indications that the overall consequences of climate change on human wellbeing and animal welfare should be negatively evaluated. Populations in the Global South and future generations are specifically vulnerable to the negative impacts of climate change.

These findings, and the hard-won scholarly consensus that pertains to them, should suffice to undercut the challenge of climate skeptics and denialists, who question that anthropogenic climate change is real or should be regarded as a ground for concern.

With that said, in recent years scientific and societal debates have increasingly shifted away from questioning the reality and seriousness of anthropogenic climate change, and moved towards assessing its regional 
impacts, as well as the desirability of different mitigation and adaptation policies. These issues are subject to various scientific and moral uncertainties, which will be discussed in this chapter.

Section 2 introduces the concept of uncertainty and distinguishes between several of its types. Section 3 discusses the main locations of uncertainty in climate science. Section 4 overviews three topics in climate ethics and economics that give rise to various uncertainties. Section 5 takes up the question of how to represent uncertainties. Section 6 outlines three general strategies for decision-making in the face of climate uncertainty. Section 7 concludes by underscoring the importance of adequate uncertainty communication to the general public and to policymakers.

\section{Conceptualizing Uncertainty}

The term uncertainty is infused with meanings. Uncertainty is typically associated with a lack of knowledge and negatively appraised, as a deficit of knowledge that should be overcome. This is only one face of uncertainty, however. Apart from what is unknown, uncertainty might also refer to what is unstable, indefinite, or indeterminate. Some species of uncertainty cannot be overcome by knowledge acquisition. Moreover, new information may serve to increase, rather than reduce, the complexity of a problem, thereby introducing new uncertainties.

Uncertainty can refer both to states of knowledge and to states of the world. It has been regarded as a measure of ignorance and as a hallmark of complexity. Hence, there are various kinds of uncertainty, and the term is conceptualized somewhat differently in different fields of inquiry. For instance, a standard definition in the study of measurement (metrology) is the following:

- Metrological definition:

The word "uncertainty" means doubt, and thus in its broadest sense "uncertainty of measurement" means doubt about the validity of the result of a measurement (JCGM 2008).

A rather different definition is found in the fields of economics and standard decision theory, where a distinction is commonly drawn between decision-making under risk and decision-making under uncertainty (Luce and Raiffa 1957). This distinction goes back to the work of the economist Frank Knight (1921), who treated risk as calculable and uncertainty is incalculable. Knight's distinction is sometimes complemented with the further category of decision-making under ignorance. If we understand probabilities in evidential terms, i.e. as the degree of evidence that pertains to a given proposition, then this leads to the following tripartite structure:

- Economical / decision-theoretical conceptualization:

- Decisions under risk involve the availability of precise evidential probabilities with high epistemic credentials;

- Decisions under uncertainty involve imprecise evidential probabilities, or evidential probabilities with low epistemic credentials;

- Decisions under ignorance involve a complete absence of decision-relevant evidence.

\subsection{A Typology of Uncertainty}

Various proposals have been advanced to articulate a typology of uncertainty which disentangles its multiple dimensions (e.g. Kwakkel et al. 2010; Smith and Stern 2011; Petersen 2012; Walker et al. 2013). Perhaps unsurprisingly, given the divergent conceptualizations of uncertainty in different fields of inquiry, no single framework is endorsed across disciplines. The variety of typologies need not be regarded as an embarrassment of riches, however: uncertainty typologies can serve different purposes, and it may be helpful to tailor typologies to specific contexts. Moreover, the typologies that have been articulated in the scholarly literature do involve clear commonalities. For instance, in one way or another, all of them distinguish at least between 1) uncertainty due to lack of knowledge; 2) uncertainty due to variability in the phenomenon of interest; 3 ) uncertainty due to ambiguity. This threefold distinction, one might say, pertains to the grounds of uncertainty. It identifies what makes it the case that we are not fully certain - i.e. what grounds our uncertainty. 
Below I outline a typology that elaborates on the grounds of uncertainty in climate policy. It does so by introducing an orthogonal distinction between uncertainty in the scientific and the moral domain. Scientific uncertainty pertains to states of the world and our knowledge and understanding of it. Moral uncertainty pertains to norms, obligations and judgements of value. The distinction is somewhat idealized: policy-decisions about climate mitigation and adaptation are typically beset with both types of uncertainty, and sometimes these are entangled (see B1). Nonetheless, it can be a useful exercise to identify what types of uncertainty are involved in a policy-decision, and to what extent these are grounded in matters of fact or value. Doing might help decisionmakers to figure out to whom to turn for expert advice (a scientist or an ethicist?), or to determine whether uncertainty can be reduced, and how so.

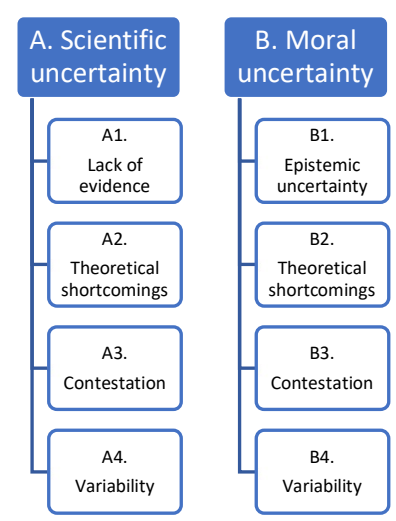

Figure 1: Grounds of scientific and moral uncertainty.

\section{A1. Scientific uncertainty grounded in lack of evidence}

Scientific uncertainties about climate change can be grounded in a lack of solid evidence. The instruments used for measuring climate change may be deficient, resulting in incomplete or biased observations. Sampling errors can lead to unrepresentative data. The models into which data are fed can be overly simplistic. The initial conditions from which computer simulations are run maybe unknown. These and other deficits yield imperfections in our body of evidence and lead to scientific uncertainty. Importantly, this uncertainty transpires in virtue of impaired observations, skewed data and inadequate scientific models, but not in virtue of the phenomena under observation. Therefore, at least in principle, evidential uncertainty can be overcome, for instance by conducting better measurements. While perhaps difficult in practice, there is no principled obstacle to eliminating evidential uncertainty.

\section{A2. Scientific uncertainty grounded in theoretical shortcomings}

Scientific uncertainty can also be grounded in more basic conceptual or theoretical deficits. Uncertainty of this kind is sometimes referred to as ambiguity. Like uncertainty, ambiguity is a term infused with meanings. In the context of the present typology, it refers first and foremost to unclarities of conceptualization and theoretical interpretation. In contexts of ambiguity, even when an appropriate amount of information is provided, it remains unclear how this information should be understood. Hence, contrary to A1-type uncertainty, A2-type uncertainty does not stem from a paucity of good evidence. It stems from a paucity of conceptual and theoretical frameworks for interpreting the evidence, or from a lack of clarity about the problem structure and the variables involved, which is generally acknowledged in the expert community. The issue is theoretically underdeveloped. Uncertainty may be overcome, but only by improving conceptual and theoretical tools.

Note that in the economics literature, ambiguity is often contrasted with the notion of risk, and taken to be synonymous with uncertainty in the decision-theoretical sense outlined at the opening of this section - i.e. as referring to situations in which probabilities cannot be determined. Indeed, contexts of ambiguity often preclude probabilistic assessment. For purposes of the present typology, however, the question of whether uncertainty can be represented in probabilistic terms had best be regarded as an orthogonal issue, which has to do with the 
representation of uncertainty (see section 5). What grounds uncertainty is not the absence of probabilities per se, but the absence of an adequate theoretical or conceptual framework of interpretation.

\section{A3. Scientific uncertainty grounded in contestation}

While some ambiguities are consensus-based (A2), other ambiguities are based on disagreement and conflict (Hansson 2018). Scientists can have a different take on an issue and there may be good arguments on either side. Rather than a shortage of theoretical frameworks, the issue may be theoretically overdetermined, with different scientists adhering to different theoretical frameworks. Peer disagreement can itself be a source of uncertainty: sometimes we do not know what to believe, because we do not know who to believe. Importantly, in discussing contested facts it is important to make a further distinction: are the facts contested among scientific experts, or are they merely contested by interest groups or among the general public, but not by scientists? These types of contestation have different epistemic implications: while expert disagreement can be a justifiable reason to lower one's credence in a scientific proposition, the same does not hold for any kind of disagreement regarding a scientific proposition.

\section{A4. Scientific uncertainty grounded in variability}

Not all scientific uncertainty transpires in virtue of human ignorance, or theoretical deficits. Uncertainty may also transpire in virtue of the variability that is inherent to the system under study. Natuaral variability is common in climate science. The Earth's climate is a complex system, which changes over time. Similarly, the evolution of human societies, whose emissions and technological capacities are key determinants of future climate change, is unpredictable in many respects. Absolute certainty about the state of the Earth's climate in the year 2100 will not be achieved until the year 2100. Causal chains to the future are still open, rendering future states of the system indeterminate. The resulting uncertainty - sometimes called aleatory uncertainty - cannot be fully eliminated. The variability of the system and the forces that influence it preclude full predictability.

There has been a long philosophical tradition, inspired by the work of Laplace, of questioning whether not all uncertainty is ultimately due to human ignorance. Hard determinists following Laplace's cue maintain that if scientists would be equipped with better epistemic capabilities, they could eliminate the uncertainty due to variability. Philosophers should bear in mind, however, that this is a stylized metaphysical doctrine, detached from modern-day scientific practice. In actual climate science, there is no indication that aleatory uncertainty can be eliminated - not even with superior tools and models. That said, in practice the distinction between uncertainty due to knowledge gaps and ambiguity (A1-3) versus uncertainty due to variability (A4) cannot always be clearly drawn. Climate change frequently confronts scientists with mixed cases, in which uncertainty results both from limited knowledge and from system variability (see section 3.2). Furthermore, scientists may not be able to tell whether knowledge gaps can be overcome by further observation and better theorizing, or whether variability is a fundamental property of the system under scrutiny. Such meta-uncertainty - uncertainty about the species of uncertainty one is dealing with - is itself an example of conceptual ambiguity.

\section{B1. Moral Uncertainty grounded in epistemic uncertainty}

In recent years ethical or moral uncertainty has become a topic of increasing scholarly interest. Like scientific uncertainties, moral uncertainties can be grouped in different types (cf. Taebi et al. 2020). One of these types roughly aligns with the scientific uncertainties discussed under A1-4: moral uncertainty may be grounded in uncertainty about matters of fact. To the extent that essential factual matters are unsettled, we may be uncertain over which course of moral action to pursue. Consider the question of how much of our mitigation resources should be devoted to Carbon Capture and Storage (CCS). One area of ethical contention is the worry that money spent on CCS diverts investments away from green energy solutions. This worry is grounded in a factual claim, but the factual claims is itself contested; the resultant epistemic uncertainty may serve to aggravate moral uncertainty. Similarly, there may be epistemic uncertainty about whether people will benefit or be put at risk because of a decision, about the identity of the people affected by decisions, or the ontological status of entities to which moral theorizing pertains (e.g. Żuradzki 2019). All of these are instances of epistemic ambiguity, which fuel moral uncertainty in turn. Note, however, that greater epistemic uncertainty does not necessarily translate to greater moral uncertainty. For instance, with regard to a very risky undertaking, a substantial extent of epistemic 
uncertainty might in fact strengthen the moral judgement that this undertaking should not be pursued (see section 4.1).

\section{B2. Moral uncertainty grounded in theoretical shortcomings}

As noted in A2 our scientific theories and conceptual frameworks may be deficient. The same holds for our moral theories and concepts. There may be moral uncertainty as to whom should be the recipients of moral duties and about how the concepts of moral evaluation should be clarified. Moral values might be vague, imprecise and may turn out to be incommensurable when spelled out. This vagueness also pertains to what the guiding moral theories and normative principles entail, and to how they should be combined. Hence, it seems to be the case that moral theory itself is underdeveloped in relevant respects. This may be particularly true for climate ethics, as leading scholars in the field have argued (see e.g. Gardiner 2011 on the 'theoretical storm').

\section{B3. Moral uncertainty grounded in contestation}

Like scientific ambiguity, moral ambiguity can come along with - and can be grounded in - moral disagreement. Consider decisions that rely on expected utility maximization versus decisions that rely on the precautionary principle (section 6), or person-affecting versus non-person affecting views in population ethics. Good arguments have been articulated on both sides, and there are ongoing academic disputes over which of these principles should be morally preferred. Similarly, there might be different legitimate interpretations of moral concepts, or different defensible prioritizations of moral values. Indeed, reasonable disagreement is widespread in ethical scholarship. Conflict might also be felt at an individual level: a moral decision-maker can feel torn about how to weigh the interests of future people against the interests of presently existing people and be at loss in formulating an overall moral judgement. Furthermore, at a metaethical level it might be argued that there simply is no truth to the matter as to how this should be done: some moral claims are inherently contestable.

\section{B4. Moral uncertainty grounded in variability}

A somewhat lesser-known species of moral uncertainty concerns the unknown unknowns of moral inquiry. New values might emerge, which could affect the moral desirability of a given course of action (van de Poel 2018). Change might also result from a different prioritization of values, or changes in our circle of moral concern. Conceivably, in the future we may come to regard all mammals as our moral equals, or we may come to think differently about the perils of existential risk. Although such changes cannot be predicted in advance, the bare fact that values might change can be anticipated. This insight, in turn, casts some degree of moral uncertainty over policy decisions, as these might have unexpected moral ramifications. For instance, our current decisions might put future groups at risk, of which we were not able to foresee their existence as morally significant entities (Hayenhjelm 2018). These kinds of unknown unknowns are most prevalent with regard to decisions that have long-term implications, as climate mitigation and adaptation decisions often do (section 4.3).

\subsection{Ground vs. Extent of Uncertainty}

The typology outlined in this section captures several faces of uncertainty, but not all of them. Further distinctions could be made, some along orthogonal dimensions. For instance, a further category frequently invoked in classifying uncertainty is 'deep uncertainty'. According to the Society for Decision-Making under Deep Uncertainty (DMDU) "deep uncertainty exists when parties to a decision do not know, or cannot agree on, the system model that relates action to consequences, the probability distributions to place over the inputs to these models, which consequences to consider and their relative importance" (cf. Lempert et al 2003). While deep uncertainty, thus understood, partly overlaps with ambiguity, the term is typically used to emphasize extent of uncertainty, rather than the ground of uncertainty. Deep uncertainties cannot be expressed in meaningful probabilistic terms and require 'non-standard' approaches to decision-making. Yet in climate change decisionmaking deep uncertainty is actually very common, and non-standard approaches are becoming increasingly prevalent (section 6).

\section{Locating Uncertainties in Climate Science}


Apart from their grounds and extent we can also categorize uncertainties by their location (Petersen 2012). Uncertainties are located at various stages of scientific enquiry and normative decision-making. This section discusses some important locations of uncertainty in climate science. Since the climate sciences span many disciplines, each of which comes along its own uncertainties, a comprehensive treatment is beyond the chapter's scope (see the chapter by Morrison in this volume for further discussion). Instead, in what follows, I will focus on three locations of scientific uncertainty in particular: observation, simulation and impact.

\subsection{Observational Uncertainties}

In the 1940s controversy arose over historical measurements of the sea temperature. The controversy was resolved when researchers realized that the bucket that had been used to measure the water's temperature had at some point been replaced, and that the same water measured in a different bucket gave rise to different measurements (Schmidt 2008). Measurement errors like these are common to practices of data collection. The quality of observed data depends on the reliability of measurements. If the equipment is ill-calibrated, measurement errors may creep in. Unfortunately, for some instruments used for climate measurements, calibration is difficult: satellites that are inaccessible once deployed cannot easily be tested and recalibrated (Baumberger et al. 2017).

There are various other ways in which measurements can be unreliable and may give rise to biased data. Observations are typically sparse in space and short in time: the sample that is obtained through observations may not be representative of large-scale trends. Historical measurements may be absent altogether, in which case scientists typically gather proxy data, which are less reliable than direct measurements. Incompleteness, potential bias and the possibility of error all add to scientists' scientific uncertainty about the quality of the observed climate data (e.g. Winsberg 2018).

Observational uncertainties can sometimes be reduced. For instance, it may sometimes be possible to compare datasets, which have been obtained by independent measurement devices. Following the insight of Pierre Duhem, however, it should be kept in mind that data are theory-laden: the calibration of measurement devices, as well as the regimentation, restructuring and subsequent interpretation of data, are influenced by auxiliary hypotheses. The theory-ladenness of observations, and the accompanying possibility of theoretical error, constitute a further source of uncertainty in measurement, which cannot be eliminated entirely.

\subsection{Simulation Uncertainties}

One major endeavor in climate science - especially the physical climate sciences - is to simulate future temperature changes and their impact on the climate system (e.g. Petersen 2012; Winsberg 2018). Computerized simulations are based on climate models, which may pertain to ocean circulations, atmospheric circulations, or couplings thereof. Several global climate models (GCMs) designed by teams of scientists around the globe are compared and put together, resulting in complex 'multimodel climate ensembles' that simulate climate change for this century and beyond. Their results provide key inputs for reports of the IPCC (Intergovernmental Panel on Climate Change).

To generate projections of future climate change these models require input about indeterminate variables - crucially, about future human greenhouse gas emissions. Hence, the projections of climate models are conditional on emission scenarios. Projections, here, should not be conflated with predictions about what is going to happen in the future: climate projections merely show how climate models respond to external forcing scenarios, and the IPCC is explicit not to attach any likelihood to these scenarios. This caveat notwithstanding, projections are important instruments for policymakers, as they do seem to provide at least some indication of what might plausibly happen in the future, depending on future human greenhouse gas emissions. Indeed, the different emission pathways outlined by the IPCC are frequently used as a basis to assess different mitigation options.

There are several kinds of uncertainties that pertain to climate simulations, which can be partitioned in different ways. Here I discuss three main classes (Hawkins and Sutton 2009): uncertainty due to the internal variability of the climate system, uncertainty regarding the external forcings of the climate system and uncertainty about climate models.

\subsubsection{Natural Variability}


The Earth's climate system exhibits substantial natural variability: fluctuations occur even in the absence of external forcings and transpire naturally from the non-linear interactions in the climate system. An example is the El Niño-Southern Oscillation, which results from the interaction between atmosphere and ocean in the tropical Pacific. Natural variability generates aleatory uncertainty, which is unlikely to be reduced even if climate models improve (Deser et al. 2012).

\subsubsection{External Forcings}

External forcings are influences on the climate system that shift the equilibrium between how much energy the Earth receives from sunlight and how much energy is radiated out. Several external forcings influence the climate system, including forcings that are anthropogenically induced. While part of the carbon dioxide humans emit remains in the atmosphere for a long time, the majority of the warming response is due to emissions caused within people's own lifetime (Ricke and Caldeira 2014). By implication, how climate change will evolve over the next decades and centuries crucially depends on the actions of current and future generations. Yet there is substantial uncertainty with regard to the amount of greenhouse gases humans will emit over the next decades and the rate with which emissions will be curtailed. Hence, there is uncertainty regarding future emissions scenarios - a type of uncertainty sometimes called 'scenario uncertainty'. This uncertainty, in turn, is bound up with questions about the future of human technology, political institutions and demography, to which we will return in section 4 . The further we look in time, the greater scenario uncertainty becomes.

Apart from uncertainty about anthropogenic forcings there is also uncertainty about the natural forcings that will influence future climates. Massive volcanic eruptions, for instance, are difficult to predict, but historical and geological evidence shows that they can be gamechangers in episodes of global warming and cooling. Solar intensity, too, can vary over time. In climate science such effects not described as being internal to the natural variability of the climate system (section 3.2.1), but as external forcings that act on the system. Hence, apart from 'internal variability' the climate system also exhibits 'external variability'.

\subsubsection{Model Response}

Even in response to the same emission scenarios, different models simulate different climate futures. This variability points to uncertainty about the accuracy of these models. Our knowledge of the climate system is limited, specifically with regard to the question of how sensitive the climate is to increased concentrations of greenhouse gas in the atmosphere. The notion of 'climate sensitivity' refers to the degrees of warming that would eventually occur if greenhouse gas concentrations were doubled from their pre-industrial baseline of 280 parts per million. Estimating climate sensitivity is complicated by the various feedback loops in the climate system. Since 1979 the commonly accepted range has been from 1.5 to $4.5^{\circ} \mathrm{C}$, which is a substantial range of uncertainty. Moreover, it has turned out to be difficult to diminish this uncertainty range in the subsequent decades, although recent work does indicate that scientists' understanding of climate sensitivity and the uncertainty that pertains to it is becoming increasingly refined (Sherwood et al. 2020).

Model uncertainty stems from various sources. Aerosols and processes of cloud formation are notoriously difficult to represent in spatial and temporal detail; their parametrization is a substantial source of uncertainty in climate models (McFarlane 2011). Another source of uncertainty is incompleteness: by representing some phenomena and neglecting others, modelers may overlook relevant processes, such as various positive and negative feedback cycles that are currently not well understood. Current models tend to assume - perhaps naively - that atmospheric feedbacks scale linearly with surface warming. Thresholds effects that are uncertain but nonetheless deemed possible by many experts, such as the collapse of the West Antartic Ice Sheet, are typically not incorporated in simulation models.

Models are inherently simplified systems. They may be adequate for some purposes and inadequate for others. While this holds true for models in general, there are some characteristics that make existing climate models specifically problematic - more so, for instance, than weather models. For instance, a crucial test for validating climate models is to evaluate them against present observations and to predict the historical record (hindcasting). The shortness of the observational record, however, constitutes a problem in doing so (Parker 2010). Furthermore, to the extent that validation is possible at all, it appears that none of today's climate models has a perfect fit with the observed record. In fact, all extant models give rise to many results that do not come close to matching observed data, beyond margins of statistical error (Baumberger et al. 2017). 
One might hope that the ensemble of models used to estimate climate sensitivity serves to balance the biases that stem from the reliance on any particular model and can help to infer which simulations are more wellfounded than others. Prima facie, it seems plausible to think that robust projections - i.e. projections that are replicated in a majority of models in the ensemble - are likely to be true. However, when it comes to model ensembles, an inference from the robustness of projections to their likely truth is problematic (Baumberger et al. 2017). Today's models have not been ensembled to span a specific uncertainty range. Moreover, the models in multimodel ensembles rely on similar assumptions and might have similar flaws, which could lead to similar projections. The combination of uncertainties at play in model ensembles, and the imperfections of bias correction measures, makes our epistemic position with regard to the resulting climate simulations somewhat opaque, and suggests that surprises are to be expected.

\subsection{Impact Uncertainties}

While computerized simulations constitute an important component of climate science, the climate sciences span a much wider field. Various environmental, social and medical sciences study the environmental and human health impacts of rising temperatures and atmospheric $\mathrm{CO}_{2}$ levels. Each of these disciplines brings along its own set of uncertainties regarding observed data and anticipated impacts. When it comes to adaptation planning in the face of anticipated environmental impacts, policymakers often find themselves in situations of even greater uncertainty than when presented with uncertainties regarding mitigation decisions. This is because uncertainties are additive: they cascade from one level to the next, leading to an ever-broadening "envelope of uncertainty" (Wilby and Dessai 2010).

Even though substantial confidence may pertain to assessments of the kinds of environmental consequences are to be expected in the face of rising temperatures, sea levels and ocean acidification, the precise details of these consequences, such as the magnitude of storms caused by sea-level rise, may be impossible to predict. The same holds for the regional distribution of climate change impacts, which is a source of substantial uncertainty. Merely focusing on changing global average temperatures conceals substantial regional variation. Techniques to downscale global climate models to regional levels are fraught with difficulties (Hewitson et al. 2014). This is unfortunate, since from the perspective of risk assessment and for purposes of adaptation planning regional differences and associated climate hazards are of distinct importance. Special mention should be made of compound risk events (e.g. heavy rain on saturated soils, or globally synchronized heatwaves affecting global food production), which amplify environmental and human impacts (Zscheischler et al. 2020). While compound events are impossible to predict in detail, the increased frequency of this type of event due to climate change is great importance for adaptation planning.

\section{Uncertainties in Climate Economics and Ethics}

There is some overlap between the key uncertainties that pertain to the climate sciences on the one hand, and uncertainties in climate economics and ethics on the other. For instance, as discussed in section 3.2.2, climate simulations crucially depend on assumptions about future greenhouse gas emissions. Emissions, in turn, depend on variables such as the development of human technologies for reducing carbon emissions and carbon uptake, the pace of the energy transition and associated political challenges, the future of demography as well as economic growth. These topics are also of great importance in climate ethics and economics. Economists, in particular, have played an important role in the development of so-called Integrated Assessment Models (IAMs) (Fleurbaey et al. 2019). These are models which incorporate the interactions between climatic changes and social developments, and which are meant to assist policymakers in making good decisions regarding climate mitigation.

In this section I single out the following three topics, each of which is couched in substantial factual as well as moral uncertainty: future technologies, future institutions and future populations. I highlight these topics as they constitute important variables which may tilt the overall balance of decision-making, for instance in the context of IAMs. However, it should be borne in mind that uncertainties in climate ethics and economics are not limited to these issues.

\subsection{Future Technologies}


Emerging technologies will play a crucial role in the transition towards a non-fossil economy. Examples include decarbonization technologies in transportation, CCS-technologies in heavy industry and advanced energy storage technologies that stimulate the deployment of renewable energy. Each of these have a clear potential to help curtailing greenhouse gas emissions, but their rate of societal uptake over the next years and decades is uncertain. Various other technological developments might serve to accelerate $\mathrm{CO}_{2}$ mitigation, although some innovations can also have mixed or opposite effects. For instance, the development of cultured meat has potential to severally reduce agricultural emissions. On the other hand, growing cultured meat is highly energy intensive; its potential effect on climate mitigation will depend on broader developments in the energy sector. Similarly, future use of artificial intelligence and blockchain might serve to exacerbate energy demands. Whatever new affordances and constraints emerging technologies generate, it is very plausible that they will have a major - yet partly unpredictable - influence on climate change mitigation and adaptation.

Somewhat more speculative, but of substantial interest to ethicists, is the emergence of geo-engineering technologies, such as carbon dioxide removal (CDR) and solar radiation management (SRM). There are different proposed ways of engaging in CDR, some of which are unconventional and risky, such as the iron fertilization of the oceans. The idea behind SRM is to block incoming sunlight by increasing the concentration of atmospheric aerosols. While this will not reduce the amount of $\mathrm{CO}_{2}$ in the Earth's atmosphere and its uptake in the oceans (therefore, it won't stop the acidification of the oceans), it could serve to revert global temperature rise. Both SRM and some of the more invasive forms of CDR come along with substantial amounts of recognized uncertainty. As a result, these technologies are ethically controversial; many ethicists think that - at least at present - they are much too risky to be deployed (e.g. Gardiner 2011). Notice, in this case, that the substantial amount of scientific uncertainty that pertains to geo-engineering does not serve to increase moral uncertainty. In fact, the opposite is the case: the amount of scientific uncertainty that comes along with deploying invasive geo-engineering technologies is what fosters the present ethical consensus to refrain from them.

\subsection{Future Institutions}

The political institutions of human societies are a source of substantial uncertainty with regard to long-term economic models. Societal ambitions regarding climate mitigation can fluctuate and democratic elections may yield surprising results. Of specific importance in the context of climate mitigation will be the ambitions of economic and demographic centers of gravity like China, India and the United States. For each of them, mitigation ambitions over the next decades are somewhat unpredictable. Moreover, the political and societal dynamics can be disrupted by unforeseen events. An example is the Covid-19 pandemic, which has had a significant impact on reducing global greenhouse gas emissions in 2020 (Liu et al. 2020). The institutional response to such events, and the institutional changes that might occur in its wake, cannot be predicted, but could be of great importance with regard to socio-economic pathways and future emissions.

Turning to normative questions of ethics and political theory, there is some uncertainty over the question of how future institutions should be designed. Does an effective global response to climate change require new forms of global governance, and if so, under what conditions would these be legitimate? Furthermore, there is some uncertainty over the question of how future people should be institutionally represented, which is related to a more fundamental uncertainty over the status of future people in moral theory (Gonzalez-Ricoy and Gosseries eds. 2016).

\subsection{Future Populations}

Human demographics are a further variable with major impact on long-term economic models. How will population growth proceed in different parts of the world, and affect global energy demands? Scientific uncertainty besets these questions, although the bounds of uncertainty are not without constraint - predictions about $20^{\text {th }}$ century population growth have been fairly stable over time. However, future populations are also a source of moral uncertainty and have raised intense disputes among climate economists (Scovronick et al. 2017). Specifically, the discount rate that economic models apply to the goods of future people has been a source of contention. Such contention is entangled with the question of how the risks and benefits of climate mitigation should be distributed across generations, and with assumptions about the future of human welfare. Given the complexity of these issues normative ambiguity cannot be easily resolved. 
Debates about discounting have a salient ethical dimension (Mintz-Woo forthcoming). If people will be better off in the future, then it may be morally justified to prioritize the interests of the present generation and to discount the interests of future people. But by what degree, if any? Is the assumption that people will be better off in the future even plausible? And how to estimate the number of future people in the first place? These questions are beset with various species of scientific and moral uncertainty. Since policy decisions about how drastically to cut carbon emissions are sensitive to these uncertainties, an important task in normative theory is to assess what are justifiable procedures for decision-making in the face of uncertainty.

\section{Representing Uncertainty}

Before we turn to the topic of decision-making under uncertainty, it is worthwhile to reflect on some of the different ways in which uncertainties can be represented. Indeed, such reflection has a certain conceptual priority: the merits of different decision-procedures under uncertainty depend, in part, on the conceptual framework used to represent uncertainty.

\subsection{Quantifying Uncertainty}

A common way to represent uncertainties is by quantifying them and expressing them probabilistically. Probability statements admit of different degrees of precision. Some statements can be framed in terms of precise probabilities - e.g. "the probability that a given climate expert endorses the scientific consensus that global warming is human caused is 97\%" (Cook et al. 2013). A greater range of uncertainty can be attached to statement couched in terms of a probability interval - e.g. "the scientific consensus on anthropogenic global warming is in the range of $90 \%-100 \% "$ (Cook et al. 2016).

Probability statements are conditional on the assumption that the underlying argument, method or model on which they are based is correct (Ord, Hillebrand and Sandberg 2010). First-order probability statements can themselves be qualified, if there is uncertainty regarding the accuracy of the underlying model. Such second-order epistemic qualification - which, too, may be expressed in probabilistic terms - is often desirable when scientific predictions derive from a particular model, the reliability of which should be assessed in its own terms. For instance, the assertion that "the probability that a given domain expert endorses the scientific consensus that global warming is human caused is $97 \%$ " is conditional on the soundness of the method employed by Cook et al. 2013. We might conceive of a climate skeptic whose confidence that this method is sound might is quite low - say only $10 \%$ - in which case the climate skeptic might still feel entitled to question whether a majority of scientists endorses the view that global warming is human caused. Judged only by standards of probabilistic rationality, there is nothing at fault with such reasoning.

There are definite upsides to presenting uncertainties in probabilistic terms. Qualitative terms such as "likely" or "improbable" can be interpreted in many different ways and may give rise to misunderstandings or conflicting interpretations. In order to rank risks and to reason precisely about degrees of evidence, numbers are indispensable (Ord 2020).

That said, the language of probabilities is not only one that can be used to represent uncertainty, and expression in qualitative terms may also pose certain advantages. While qualitative representation often comes at a loss of precision, it may lead to a gain in faithfulness, if the uncertainty is such that even probability estimates cannot be reliably made. Also, in some instances, natural language might actually convey more clearly whether or not a probability is decision-relevant ("this chance is non-negligible"), whether probabilistic language is more open to interpretation (is a chance of 1 in 100 non-negligible? 1 in 1000? 1 in 10000?).

Moreover, not all kinds of uncertainty lend themselves for probabilistic representation. For instance, there is no straightforward method to cast uncertainties due to ambiguity in probabilistic terms. This puts some pressure on efforts to rank contexts of knowledge in terms of the extent of uncertainty they involve, going from complete certainty, uncertainty that involves precise probabilities, uncertainty that involves vague probabilities, deep uncertainty in which probabilities are inherently contested, all the way down to total ignorance (Walker et al 2013). Such a ranking of uncertainty may be somewhat misleading, however, as it identifies the extent of ignorance with the precision that probabilistic statements allow for. Yet some types of uncertainties are genuinely different in kind, which precludes any straightforward kind of ranking. Which means of representing uncertainty 
- probabilistic or not - is most suitable depends not only on the extent of uncertainty, but also on the type of uncertainty in question (see section 2).

Apart from conceptual reflection, when it comes to the effectiveness of different kinds of uncertainty representations psychological research is of great importance. Recent work suggests that in communicating climate uncertainty there is a delicate balance to be struck between being too precise and overly vague (Ho and Budescu 2019). While decision-relevant details should not be left out in uncertainty communication, efforts to be too comprehensive may backfire. Moreover, it is important to use terminology that puts clear bounds on the uncertainties being communicated (e.g. by specifying a specific range of sea-level rise, rather than specifying that the range is unknown). Bounded uncertainty statements are more easily accepted among the public, whereas uncertainty statements that admit to a lack of resolution decreases confidence in the statement (Howe et al. 2019).

\subsection{The IPCC's Treatment of Uncertainty}

The IPCC employs a standardized vocabulary to represent uncertainties, meant to communicate uncertainty in a clear and consistent manner. The framework that has been in place during the fifth assessment cycle is outlined in Mastrandrea et al. 2010. The IPCC's "calibrated language" contains two key metrics for communicating the degree of uncertainty of a statement: confidence and likelihood. Confidence is expressed in qualitative terms (e.g. "high confidence"). Levels of confidence are a function of the type and quality of evidence on the one hand, and degree of agreement on the other. Hence, if an assertion is qualified with "high confidence", then we can infer that there is solid evidence and substantial scientific consensus regarding its truth.

The IPCC's likelihood scale contains quantified measures of uncertainty presented in terms of likelihood intervals for relevant variables. Likelihoods are based on statistical analysis of observations or model results, or on expert judgment. For instance, the fifth assessment report states that it is "extremely likely" that more than half of the late twentieth century warming was human caused (IPCC 2013). Since the IPCC uses the term "extremely likely" to express an probability interval of $95-100 \%$, we can infer that climate models paired with expert judgement give us a $95-100 \%$ likelihood that a majority of the temperature rise that occurred during the last century has been human induced.

\subsection{Scenarios, Narratives, Storylines}

In the wake of AR5 increasing criticisms regarding the IPCC's representation of uncertainty have been voiced, as it seems to underplay the severity of the risks of global warming. (e.g. Stoerk et al. 2018; Herrando-Pérez 2019). For one, it has been questioned whether the terminological conventions the IPCC employs convey the right message to the public and to decision-makers. Arguably, the IPCC's terminology has been too conservative and occasionally vague: reliability is achieved at the cost of informativeness (Løhre et al. 2019) Moreover, it has been questioned whether the IPCC's treatment of uncertainty is not too probability oriented. Arguably, given the range of uncertainties that pertain to climate science (see section 3), for many statements about the magnitude of global warming, let alone for statements about its regional impacts, it is questionable whether meaningful probability statements - and even vague probability estimates - can be attached to them (Sutton 2019). This is not to say, however, that we do not have any relevant information with regard to these magnitudes and impacts, or that such information is not relevant for decision-makers. To the contrary, in the context of risk assessment and adaptation planning "low likelihood high impact events" are of the utmost importance. The general tenor of the criticism is that the IPCC's approach should be better aligned with key principles of risk assessments (King et al. 2015), by focusing on precisely these types of events. There are indications that in AR6, which will be published around the same time as the present chapter, the IPCC approach will shift accordingly, by adopting a "disaster risk reduction" framework (Sillmann et al. 2021).

Partly borne out of a dissatisfaction with the dominant approaches to modelling climate futures and representation of risks, in recent years increasingly scholarly attention has been paid to approaches that have a less quantitative focus - i.e. so-called scenarios, narratives, or storylines. These are explorative tools, which do not predict what will happen, but aim to sketch realistic possibilities of what might happen. Shepherd et al. (2018) characterize a storyline as a physically self-consistent unfolding of past events or of plausible future events, to which no specific probability is attached. Storylines are intended to provide information that is particularly well suited for political decision-makers. At a psychological level, their built-in narrative enhances emotional engagement. From a risk perspective, they help to focus attention on compound risks which could have major 
impact, but are not captured in global climate models (see section 3.3). Furthermore, the logic of research questions that guides the storyline approach implies that it tends to safeguard against Type 2 errors - i.e. false negatives. That is to say, it tends to avoid missed warnings and ensure that the impacts of climate change are not overlooked. Arguably this is justified: climate science should become more like medicine or public health in its assessments of risk, where false alarms tend to be preferred over failures to prevent (Lloyd \& Oreskes 2018).

\section{Decision Strategies in the Face of Uncertainty}

It is commonly assumed that decision-makers should seek to eliminate or maximally reduce uncertainty. This is not always feasible, however: some uncertainties simply cannot be overcome. Moreover, the aspiration to maximally reduce uncertainty may not always have desirable consequences. Uncertainty may be an indicator of the complexity of an issue; disregarding this complexity might have the unwanted consequence of impoverishing one's decisions.

But there are other ways of coping with uncertainty, apart from seeking to reduce it. Indeed, traditional idealized guidelines for decision-making in the face of uncertainty are increasingly giving way to 'non-standard' frameworks and approaches, such as adaptive decision-making. In this section I do not aspire to a comprehensive overview, but single out three general strategies of decision-making in the face of uncertainty (cf. Hinkel et al. 2019). These need not be looked at as rivalling strategies, at least not in all contexts. Instead, they can be regarded as strategies that might be suitable in different decision-context, depending on the nature and the degree of uncertainty that confronts decision-makers.

\subsection{Expected Utility Maximization}

Proponents of expected utility theory, or expected value theory, counsel to take decisions by conducting a consequentialist type of risk-benefit analysis. The expected utility for an action is the sum of the probabilityadjusted utility of the possible consequences of an action. Maximizing expected utility is the default approach to 'decision-making under risk' - i.e. in contexts where decision-makers can assign high-credential probabilities to different outcomes (section 2). While such contexts dominate textbook examples, however, clear-cut cases of decision-making under risk are unusual in real life decision-making (Hansson 2009). When probabilities are not readily available, or can only be estimated with substantial margins of uncertainty, reliance on expected utility theory is controversial (e.g. Bradley et al. 2020). Some proponents advise to stick with the expected utility approach and try to estimate probabilities as well as possible (Broome 2012). Critics might argue that in contexts of deep uncertainty this either invites false precision, or leads to paralysis in decision-making, as there is no expert consensus regarding the appropriate probability estimates (Hopster forthcoming).

\subsection{Robust Decision-making}

The aim of robust decision making is to define policies that yield acceptable outcomes across a wide range of plausible future states of the world (Walker et al. 2013). Hence, in the case of climate change, acceptable outcomes should be robust to the range of different scenarios that might transpire in virtue of the various scientific uncertainties we face (section 3). This strategy might conflict with the strategy of expected utility maximization, which is generally biased in favour of realizing (or preventing) outcomes that are particularly likely to occur.

There are different types of robustness strategies. One of them is to outline a static policy that will perform well in practically all conceivable situations. A weakness of this strategy is the potential mismatch between what is conceivable and what is realistically possible: human imagination is limited and likely to be tainted by various cognitive biases. A second robustness strategy is to seek resilience, which may roughly be defined as a system's ability to recover from external disturbances. Resilient decision-making counsels to follow a policy such that whatever happens in the future, a given system should be able to quickly recover. A third type of robustness approach is to plan specifically for the worst possible case. This approach is sometimes associated with the precautionary principle, although it should be kept in mind that the precautionary principle has been rendered in many different ways, not all of which are specifically geared at avoiding the worst outcome. An element that many renderings have in common is a reliance on a decision tripod, which consists of a damage condition, an epistemic condition and a proposed remedy (Steel 2014). Precautionary decisions may be defensible if the envisioned damage is great, its occurrence constitutes a realistic possibility, and the remedy is effective in preventing this possibility from actualizing. 


\subsection{Adaptive Decision-making}

A third family of decision-making counsels to devise dynamic policies that can change over time and are adaptive to the challenges at hand. Given that the rate of future climate change is couched in substantial uncertainty, and given that human societies and technologies may evolve in unforeseen directions (section 4), it is seems desirable to design policies that can easily be updated when new information becomes available and that allow for flexible manoeuvring in the face of novel affordances and constraints. Adaptive planning policies are designed from the outset to respond to how the future unfolds, how the risk landscape changes and how societal values evolve (Taebi et al. 2020).

The adaptive planning framework is partly compatible with expected utility maximization. Yet it involves something of a paradigm shift from planning based on expectations to planning conditional on observed developments (Kwakkel and Haasnoot 2019). Decision-making is regarded as an experimental learning process. Especially in contexts with clear potential for unrecognized ignorance, the flexibility than the adaptive decisionmaking framework nurtures seems particularly beneficial.

\section{Conclusions}

In the face of the uncertainties that beset climate science, ethics and economics, it might appear that taking climate policy-decisions, which holistically incorporates considerations from each of these domains, is extremely daunting. This is certainly true. But as I will underline in conclusion, there are two ways in which scientists, ethicists and economists can substantially ease the task of decision-makers: by filtering uncertainty information that is policy-relevant, and by adequately communicating uncertainties.

First, not all uncertainties that matter to scientists are equally relevant for policymakers (Hansson 2018). Climate experts involved in policy-advice have a moral responsibility to be selective in their reporting of uncertainty and to emphasize those uncertainties that are specifically relevant in tilting the overall balance evidence. Apart from recognized ignorance (known unknowns), special attention should thereby be paid also to include unrecognized ignorance (unknown unknowns). For instance, given the natural variability of the climate system as well as the limitations of our understanding of this system (section 3), we know that in climate science - more so than in many other scientific disciplines - surprises are to be expected (Parker and Risbey 2015). Indeed, in virtue of the possibility of surprises, much greater epistemic humility might be warranted than a mere focus on recognized ignorance suggests.

Secondly, the question of how uncertainty is represented and communicated is of great importance. Uncertainty communication should be adequate for the purpose such communication is meant to serve (Keohane et al. 2014). Since much of climate decision-making is an exercise in risk management, uncertainty reports should be sensitive to the requirements of risk analysis (King et al. 2015). This includes filing complete reports: taking into account all the significant sources of uncertainty and the available evidence. For instance, returning to a distinction made in section $\mathbf{5}$, it would be misleading to report only first-order probabilities, if the second-order credentials of these probabilities are rather low. Instead, it is more expedient to highlight the all-things-considered balance of evidence, which might yield the insight that there is actually substantial uncertainty regarding an issue. If there is not much we can positively assert regarding the prospect of a given catastrophe, but the catastrophe cannot be excluded as a realistic possibility, then this should be the key message of the uncertainty report.

There are various further avenues for philosophical work on the topic of climate uncertainty. At a conceptual level, progress can be made by further teasing apart the several dimensions of uncertainty, as well as by articulating a more fine-grained uncertainty vocabulary, especially at the levels of deep uncertainty and ambiguity. At a normative level, there is ample to further develop and refine decision-strategies in the face of deep uncertainty. Conceptual and normative work might also be combined, for instance in attempts to conceptually ameliorate the IPCC's uncertainty terminology. Last but not least, the topic of moral uncertainty has 
only been recently taken up as a standalone topic of philosophical inquiry. Future work on this topic, and the theoretical innovations it might induce, will benefit the fields of climate ethics and politics. ${ }^{1}$

\section{Cross References}

Morrison M. Climate Change and Scientific Uncertainty. In: G. Pellegrino and M. Di Paolo, eds. 2021.

Handbook of the Philosophy of Climate Change.

\section{References}

Baumberger C., Knutti R. and Hirsch Hadorn G. 2017. Building Confidence in Climate Model Projections: An Analysis of Inferences from Fit. WIREs Clim. Change 8, e454. doi: 10.1002/wcc.454

Bradley R., Frigg R., Steele K., Thompson E. and Werndl C. 2020. The Philosophy of Climate Science. Internet Encyclopedia of Philosophy. https://iep.utm.edu/climate/

Broome J. 2012. Climate Matters: Ethics in a Warming World. New York: Norton.

Cook J., Oreskes N., Doran P.T., et al. 2016. Consensus on Consensus: A Synthesis of Consensus Estimates on Human-caused Global Warming. Environ. Res. Lett. 11 048002. https://doi.org/10.1088/1748-9326/11/4/048002

Cook J., Nuccitelli D., Green S.A., Richardson M., Winkler B., Painting R., Way R., Jacobs P. and Skuce A. 2013. Quantifying the Consensus on Anthropogenic Global Warming in the Scientific Literature. Environ. Res. Lett. 8024024.

Deser C., Knutti R., Solomon S., Phillips A.S. 2012. Communication of the Role of Natural Variability in Future North American Climate. Nature climate change 2(11): 775-779.

Fleurbaey M., Ferranna M., Budolfson M., Dennig F., Mintz-Woo K., Socolow R., Spears D., Zuber S. 2019. The Social Cost of Carbon: Valuing Inequality, Risk, and Population for Climate Policy. The Monist 102 (1): 84-109. https://doi.org/10.1093/monist/ony023

Gardiner, S.M. 2011. A Perfect Moral Storm. New York: Oxford University Press.

Gonzalez-Ricoy I. and Gosseries A. eds. 2016. Institutions for Future Generations. Oxford: Oxford University Press.

Hansson S.O. 2009. From the Casino to the Jungle. Dealing with Uncertainty in Technological Risk Management. Synthese 168: 423-432.

Hansson, S.O. 2018. Risk, Science and Policy. A Treacherous Triangle. Ethical Perspectives 25(3): 391-419.

Hawkins E. and Sutton, R. 2009. The Potential to Narrow Uncertainty in Regional Climate Predictions. Bulletin of the American Meteorological Society 90(8): 1095-1107.

Hayenhjelm M. 2018. Risk Impositions, Genuine Losses, and Reparability as a Moral Constraint. Ethical Perspectives 25(3): 419-446.

Herrando-Pérez S., Bradshaw C.J.A., Lewandowsky S., Vieites, D.R. 2019. Statistical Language backs Conservatism in Climate-Change Asessements. BioScience 69(3): 209-219.

https://doi.org/10.1093/biosci/biz004

Hewitson B.C., Daron J., Crane R.G., Zermoglio M. F. and Jack C. 2014. Interrogating Empirical-statistical Downscaling. Climatic Change 122: 539-554. DOI 10.1007/s10584-013-1021-z

\footnotetext{
${ }^{1}$ I thank Lukas Meyer, Kian Mintz-Woo, Thomas Pölzler, Jonathan Knutzen, Daniel Petz, Elias Moser and Romina Rekers fur their helpful suggestions. This chapter was written with the generous support of an NWO Rubicon Grant.
} 
Hinkel J., Church J.A., Gregory J.M., Lambert E., Le Cozannet G., Lowe J., McInnes K.L., Nicholls R.J., Pol T.D., van de Wal R. 2019. Meeting User Needs for Sea Level Rise Information: A Decision Analysis Perspective. Earth's Future 7(6804): 320-337. https://doi.org/10.1029/2018EF001071

Ho E.H. and Budescu, D.V. 2019. Climate Uncertainty Communication. Nature Climate Change 9: 802-803. https://doi.org/10.1038/s41558-019-0606-6

Hopster, J.K.G. (forthcoming). Climate Uncertainty, Real Possibilities and the Precautionary Principle. Erkenntnis.

Howe L., MacInnis B., Krosnick J., Markowitz E.M. and Socolow, R. 2019. Acknowledging Uncertainty Impacts Public Acceptance of Climate Scientists' Predictions. Nat. Clim. Change 9: 863-867. https://doi.org/10.1038/s41558-019-0587-5

IPCC. 2013. Climate Change 2013: The Physical Science Basis. Contribution of Working Group I to the Fifth Assessment Report of the Intergovernmental Panel on Climate Change. Cambridge and New York: Cambridge University Press.

Joint Committee for Guides in Metrology (JCGM). 2008. Guide to the Expression of Uncertainty in Measurement, JCGM 100:2008 (GUM 1995 with minor corrections).

http://www.bipm.org/utils/common/documents/jcgm/JCGM_100_2008_E.pdf

Keohane R.O., Lane M., Oppenheimer M. 2014. The Ethics of Scientific Communication under Uncertainty. Politics, Philosophy \& Economics 13: 343-368. https://journals.sagepub.com/doi/full/10.1177/1470594X14538570

King D., Schrag D., Dadi Z., Qui Y., and Ghosh, A. 2015. Climate Change: A Risk Assessment. Cambridge University Centre for Science and Policy Rep.

Knight F. 1921. Risk, Uncertainty and Profit. Boston: Houghton Mifflin.

Kwakkel J.H. and Haasnoot M. 2019. Supporting DMDU: A Taxonomy of Approaches and Tools. In: V.A.W.J. Marchau, W.E. Walker, P.J.T.M. Bloemen, S.W. Popper (eds.). Decision Making Under Deep Uncertainty: From Theory to Practice Springer: 355-374

Kwakkel J.H., Walker W.E. and Marchau V.A.W.J. 2010. Classifying and Communicating Uncertainties in Model-based Policy Analysis. International Journal of Technology, Policy and Management 10(4): 299-315. DOI:10.1504/IJTPM.2010.036918

Lempert R.J., Popper S.W. and Bankes S.C. 2003. Shaping the Next One Hundred Years: New Methods for Quantitative Long-term Strategy Analysis (MR-1626-RPC). Santa Monica, CA: The RAND Pardee Center.

Liu, Z. Ciais P., Deng Z. et al. 2020. Near real-time Monitoring of Global CO2 Emissions reveals the Effect of the Covid-19 Pandemic. 2020. Nature Communications 11, 5172. https://doi.org/10.1038/s41467-020-18922-7

Lloyd E.A., and Oreskes, N. 2018. Climate Change Attribution: When Is It Appropriate to Accept New Methods? Earth's Future 6. https://doi.org/10.1002/2017EF000665

Løhre E., Juanchich M.,Sirota M., Teigen K.H., Shepherd T.G. (2019). Climate Scientists’ Wide Prediction Intervals May Be More Likely but Are Perceived to Be Less Certain. Weather, Climate and Society 11. DOI: 10.1175/WCAS-D-18-0136.1

Luce R.D. and Raiffa H. 1957. Games and Decisions. New York: Wiley.

Mastrandrea M.D., Field C.B., Stocker T.F. et al. 2010. Guidance Note for Lead Authors of the IPCC Fifth Assessment Report on Consistent Treatment of Uncertainties. Intergovernmental Panel on Climate Change (IPCC).

McFarlane N. (2011). Parameterizations: representing key processes in climate models without resolving them. WIREs Climate Change 2. https://doi.org/10.1002/wcc.122 
Mintz-Woo, K. (Forthcoming). A Philosopher's Guide to Discounting. In T. McPherson, M. Budolfson, \& D. Plunkett (Ed.), Philosophy \& Climate Change . Oxford, Oxford University Press.

Ord T. 2020. The Precipice: Existential Risk and the Future of Humanity. New York: Hachette Books.

Ord T., Hillerbrand R., Sandberg A. 2010. Probing the Improbable: Methodological Challenges for Risks with Low Probabilities and High Stakes. Journal of Risk Research 13: 191-205.

Oreskes N. and E.M. Conway. 2010. Merchants of Doubt. Bloomsbury Press.

Parker W.S. 2010. Predicting Weather and Climate: Uncertainty, Ensembles and Probability. Stud Hist Philos Mod Phys 41: 263-272.

Parker W.S. and Risbey J.S. 2015. False Precision, Surprise and Improved Uncertainty Assessment. Philosophical Transactions Royal Society A, 373 (20140453).

Petersen A.C. 2012. Simulating Nature: A Philosophical Study of Computer-Simulation Uncertainties and Their Role in Climate Science and Policy Advice. Boca Raton, Florida: CRC Press.

Poel, van de I. 2018. Design for Value Change. Ethics and Information Technology. DOI:10.1007/s10676-0189461-9

Ricke K.L. and Caldeira K. 2014. Maximum Warming occurs about One Decade After a Carbon Dioxide Emission. Environ. Res. Lett. 9124002.

Schmidt, G.A. 2008. Of Buckets and Blogs. RealClimate. www.realclimate.org/index.php/archives/2008/06/ofbuckets-and-blogs/

Scovronick N., Budolfson M.B., Dennig F., Fleurbaey M., Siebert A., Socolow R.H., Spears D., Wagner F. 2017. Population, Ethics, and Climate Change Policy. Proceedings of the National Academy of Sciences 114(46): 12338-12343. DOI: 10.1073/pnas.1618308114

Shepherd T.G., Boyd E., Calel R.A. et al. 2018. Storylines: An Alternative Approach to Representing Uncertainty in Physical Aspects of Climate Change. Climatic Change 151: 555-571.

https://doi.org/10.1007/s10584-018-2317-9.

S.C. Sherwood, M. J. Webb, J. D. Annan, K. C. Armour, P. M. Forster, J. C. Hargreaves, G. Hegerl, S. A. Klein, K. D. Marvel, E. J. Rohling, M. Watanabe, T. Andrews, P. Braconnot, C. S. Bretherton, G. L. Foster, Z. Hausfather, A. S. von der Heydt, R. Knutti, T. Mauritsen, J. R. Norris, C. Proistosescu, M. Rugenstein, G. A. Schmidt, K. B. Tokarska, and M. D. Zelinka. 2020. An Assessment of Earth's Climate Sensitivity Using Multiple Lines of Evidence. Reviews of Geophysics 58, e2019RG000678.

https://doi.org/10.1029/2019RG000678

Sillmann, J., Shepherd, T. G., van den Hurk, B., Hazeleger, W., Martius, O., Slingo, J., \& Zscheischler, J. 2021. Event-based storylines to address climate risk. Earth's Future, 9, e2020EF001783.

https://doi.org/10.1029/2020EF001783

Smith L.A. and Stern N. 2011. Uncertainty in Science and its Role in Climate Policy. Phil. Trans. R. Soc. A 369: 4818-4841.

Steel D. 2014. Philosophy and the Precautionary Principle. Cambridge: Cambridge University Press.

Stoerk T., Wagner G., Ward R.E.T. 2018. Policy Brief-Recommendations for Improving the Treatment of Risk and Uncertainty in Economic Estimates of Climate Impacts in the Sixth Intergovernmental Panel on Climate Change Assessment Report. Review of Environmental Economics and Policy 12: 371-376. https://doi.org/10.1093/reep/rey005

Sutton, R.T. 2019. Climate Science Needs to take Risk Assessment Much More Seriously. Bulletin of the American Meterological Society 100(9): 1637-1642. 
Taebi B., Kwakkel J.H., Kermisch C. 2020. Governing Climate Risks in the face of Normative Uncertainties. WIREs Climate Change. https://doi.org/10.1002/wcc.666

Walker W.E. Lempert R.J. and Kwakkel, J.H. 2013. Deep Uncertainty. In: S.I. Gass and M.C. Fu (eds.). Encyclopedia of Operations Research and Management Science, 3rd ed., New York: Springer: 395-402.

Wilby, R. and Dessai, S. 2010. Robust Adaptation to Climate Change. Weather 65(7):180-185. DOI: $10.1002 /$ wea.543

Winsberg, E. 2018. Philosophy and Climate Science. Cambridge: Cambridge University Press.

Zscheischler J., Martius O., Westra S. et al. 2020. A Typology of Compound Weather and Climate Events. Nat Rev Earth Environ 1: 333-347. https://doi.org/10.1038/s43017-020-0060-z. https://doi.org/10.1038/s43017-020$\underline{0060-\mathrm{Z}}$

Żuradzki, T. 2019. The Normative Significance of Identifiability. Ethics and Information Technology 21: 295305. https://doi.org/10.1007/s10676-018-9487-z 